\title{
On the Benefits of Polarization for Fixed Area Television White Space Devices
}

\author{
Tim W. C. Brown, Member, IEEE, Alex C. H. Tay
}

\begin{abstract}
The frequency spectrum offered by television white spaces provides an attractive solution to supplying extra bandwidth within large wireless local area networks or wireless backhaul links. However, it is still necessary to ensure for deployment purposes that the primary users, broadcasting services in this case, do not interfere with the white space devices but more importantly that the white space devices do not interfere with the television receivers or television retransmitters. Given the increased use of vertical polarization as opposed to horizontal polarization in digital television transmission, this paper investigates the benefits of using horizontal as opposed to vertical polarization for white space devices operating within a restricted area. For white space devices such as access points or backhaul links placed at a high height, use of horizontal polarization will reduce their vulnerability to interfere with the primary user regardless of what polarization it has, while also they will be less susceptible to interference from the primary user in locations where vertically polarized television transmitters are deployed.
\end{abstract}

Index Terms-TVWS, Path Loss, Polarization, Uda-Yagi antennas

\section{INTRODUCTION}

$\mathrm{T}$ ELEVISION white spaces (TVWSs) are an attractive means to help provide for the increasing demand for extra spectrum in wireless communication devices [1]. Digital broadcasting channels operating between $465 \mathrm{MHz}$ and $860 \mathrm{MHz}$ in the United Kingdom and at similar ultra high frequency (UHF) bands in other parts of the world can provide a range of white spaces in a given location. This allows the opportunity to use low cost radios in a white space device (WSD), which require being automatically tuned to the appropriate frequency depending on its geographical location. In the context of the scope of this paper, a white space device could include a mobile terminal device within a wireless local area network (WLAN) or small cell as well as the access point, while also it could include a wireless backhaul link [2][4]. The use of TVWS already has a commercial application in WLANs designed to operate over a larger area than conventional WLANs such that they are often termed as "Super WiFi" potentially working over a range as long as

Manuscript received October 13, 2012.

T. W. C. Brown is with the Centre for Communication Systems Research, University of Surrey, Guildford, Surrey, GU2 7XH, UK. Tel: +44 1483 689810, e-mail: tim@brown76.name.
$200 \mathrm{~m}$ [2]. Such a system would be beneficial in locations such as railway stations, airports and congress centers, where demand for WLAN usage is high and suitable bandwidth is provided for the degree of traffic. In all these cases addressed, the WSDs are operating within a fixed area be they stationary or mobile and they will be tuned to suitable white spaces based upon their location.

For a wireless system to be deployed within a fixed geographical location, it is necessary that suitable frequencies are selected in order to ensure three important conditions are met:

1. The primary user (also known as the incumbent), which are broadcasting transmitters in this case should not interfere with the secondary user, which is the WSD.

2. The secondary user must, more importantly, not cause harmful interference to the primary user. In this case, the primary user includes both television receivers, which could be connected to an Uda-Yagi antenna on top of a building, as well as a television re-transmitter, which would use an array of Uda-Yagi antennas to receive. It is necessary to model the path loss in a suitable way such that it can be ensured there is a minimum path loss from a WSD, which will not cause harmful interference to the primary user. This is ascertained by ensuring the interference does not exceed the bounds of the protection ratio of the primary user, defined as the minimum tolerable signal to interference and noise ratio [5].

3. Two or more secondary users may be in proximity to each other, in which case interference between the devices must also be minimized by means of suitable resource allocation schemes.

The third point is less important than the first two as it is expected the secondary users would have to manage each other to some extent by using a geo-location database, from which it could ascertain unused channels in a fixed location [6]-[8]. The usage of geo-location databases do have limitations and the need for augmentation using spectrum sensing techniques have been identified [9]-[14], whereby the usage of a particular frequency in broadcasting should be detected by the WSD with a power level as low as $-114 \mathrm{dBm}$ in the United States and as low as $-126 \mathrm{dBm}$ in the United Kingdom depending on the scenario [13]. This will better ascertain the vacancy of a channel. 
A geo-location database requires reliable information about the transmitted channels from the primary user and the risk to the primary user from a fixed location, much of which is dependent on using reliable path loss models. One important consideration, which has not been included in geo-location databases is the effect of polarization. This is equally important when considering co- and cross-polarization issues in spectrum sensing, which could cause difficulty if the WSD's antenna polarization is badly orientated.

Considering interference, if the majority of television transmitters are using vertical polarization as opposed to horizontal, it is highly questionable whether lower interference will result both ways if the WSD is transmitting and receiving with horizontal polarization, while spectrum sensing in both polarizations. Regardless of the polarization of the primary user, it is shown in this paper how horizontal polarization can increase the path loss from a fixed WSD to the primary user and therefore increase the size of area over which the WSD can operate or the allowed transmit power. Such results would therefore present a case for deploying horizontal polarization in WSDs but also the need for including polarization information in geo-location databases and giving consideration to polarization in spectrum sensing.

Section II of this paper will show the benefits of using horizontal polarization from the perspective of interference from the primary to secondary user. Section III will include measurements of typical Uda-Yagi antennas used in broadcasting and their cross polarization vulnerabilities, particularly with leaky feeders. Finally before the conclusion in section V, section IV will provide analysis of the expected path loss characteristics for different polarizations based on indoor and outdoor measurements with Uda-Yagi antennas.

\section{PRIMARY TO SECONDARY USER INTERFERENCE MEASUREMENTS}

A measurement campaign was set up to characterize the TVWSs across the whole UK band for digital broadcasting from $465 \mathrm{MHz}$ through to $860 \mathrm{MHz}$ using a handheld spectrum analyzer and a set of suitable antennas in order to analyze the differences in received power when the polarization is adjusted. The resolution and video bandwidths were set to $10 \mathrm{kHz}$ so as to provide a suitably low enough noise floor and hence a suitably high measurement signal to noise ratio when comparing co- and cross-polarizations. This allowed the spectrum analyzer to have a sufficiently low sensitivity while also maintaining a fast sweep time of less than 1 second for measurement purposes. The measurements were undertaken during July 2011 in three outdoor locations in Guildford, United Kingdom, which is south west of London and within range of several television transmitters in South London and within the counties of Surrey and Sussex.

Table 1 shows a list of transmitters within $25 \mathrm{~km}$ non obstructed range of the locations measured in Guildford including the channels they use, their transmit power and their polarization. The channel numbers represent different center frequencies in the UHF spectrum, where each channel has a bandwidth of $8 \mathrm{MHz}$ with guard bands. Note that at the time of measurement, the television transmissions in the area were in transition to full digital switch over and thus many of the frequencies listed are now obsolete. This does not, however, affect the validity of the results presented in this paper comparing polarizations. The vast majority of transmitters, 16 out of 21, which are lower power (and remain this way beyond switch over) are designed to broadcast over a shorter range and are vertically polarized while the remaining five are horizontally polarized. Two out of these five are considered main transmitters, high in height and designed to broadcast over a wide area, which typically require high power slotted waveguide antennas transmitting horizontal polarization [15].

Table 1 - Table showing the broadcast transmit channels, polarization and power levels within $25 \mathrm{~km}$ of Guildford. Source British Broadcasting Corporation (BBC) ${ }^{1}$

\begin{tabular}{|c|c|c|c|c|c|c|}
\hline Location & \multicolumn{4}{|c|}{ Channels } & Pol & Power \\
\hline Crystal Palace & 26 & 33 & 23 & 30 & $\mathrm{H}$ & $1000 \mathrm{~kW}$ \\
\hline Midhurst & 61 & 55 & 58 & 68 & $\mathrm{H}$ & $100 \mathrm{~kW}$ \\
\hline Dorking $\mathrm{H}$ & 51 & 44 & 41 & 47 & $\mathrm{H}$ & $55 W$ \\
\hline Old Coulsdon & 48 & 64 & 45 & 66 & $\mathrm{H}$ & $6 \mathrm{~W}$ \\
\hline Sutton & 55 & 62 & 59 & 65 & $\mathrm{H}$ & $9 \mathrm{~W}$ \\
\hline Guildford & 40 & 46 & 43 & 50 & V & $10 \mathrm{~kW}$ \\
\hline Reigate & 57 & 63 & 60 & 53 & V & $10 \mathrm{~kW}$ \\
\hline Woolwich & 57 & 63 & 60 & 67 & V & $630 \mathrm{~W}$ \\
\hline East Grinstead & 40 & 56 & 46 & 59 & V & $117 \mathrm{~W}$ \\
\hline Mickleham & 61 & 55 & 58 & 68 & V & $100 W$ \\
\hline Caterham & 55 & 62 & 59 & 65 & V & $35 \mathrm{~W}$ \\
\hline Croydon & 49 & 56 & 52 & 67 & V & $33 \mathrm{~W}$ \\
\hline Wonersh & 48 & 65 & 52 & 67 & V & $25 \mathrm{~W}$ \\
\hline Haslemere & 22 & 28 & 25 & 32 & V & $15 W$ \\
\hline Greenwich & 56 & 50 & 52 & 48 & V & $15 W$ \\
\hline Orpington & 55 & 62 & 59 & 66 & V & $15 W$ \\
\hline Dorking V & 51 & 44 & 41 & 47 & V & $14 W$ \\
\hline Biggin Hill & 45 & 52 & 49 & 67 & V & $12 W$ \\
\hline Hammersmith & 48 & 62 & 59 & 65 & V & $10 \mathrm{~W}$ \\
\hline Sutton & 55 & 62 & 59 & 65 & V & $9 \mathrm{~W}$ \\
\hline Micklefield & 54 & 64 & 57 & 67 & V & $8 W$ \\
\hline
\end{tabular}

Three chosen measurement locations in Guildford were selected and are named and described as follows:

- Cathedral - Guildford's main cathedral is on the outskirts and based upon one of the highest hills in the town, thus it is well positioned to pick up transmitted television signals from a wide area, where a WSD access point may be in such a situation. There is also a line of sight between the position outside the cathedral and the nearest television transmitter obstructed by vegetation. This is illustrated in Figure 1 (a) where the picture is taken outside the front entrance to the cathedral to the left of the picture.

\footnotetext{
${ }^{1}$ www.bbc.co.uk
} 
- Stoke Park - An open parkland area situated near to the center of the town, approximately $1.5 \mathrm{~km}$ away from the nearest transmitter shown in Figure 1 (b). The location is again on a high point, where several television signals could be received over a wide area.

- North Street - This is one of the main shopping streets based in the center of Guildford, shown in Figure 1 (c). There is not a direct line of sight to the transmitter in this instance, thus the blockages from buildings are likely to create more open white spaces compared to the other two locations with an access point based in a street.

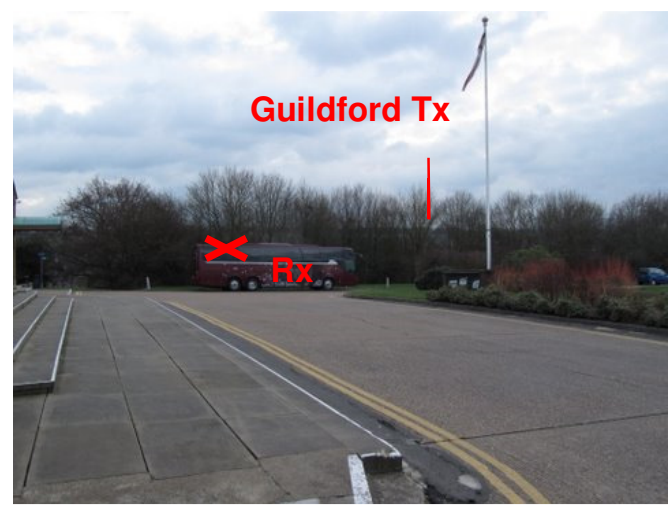

(a)

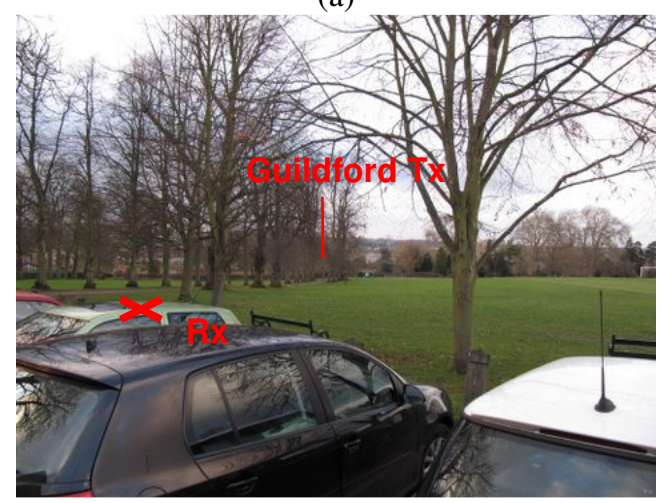

(b)

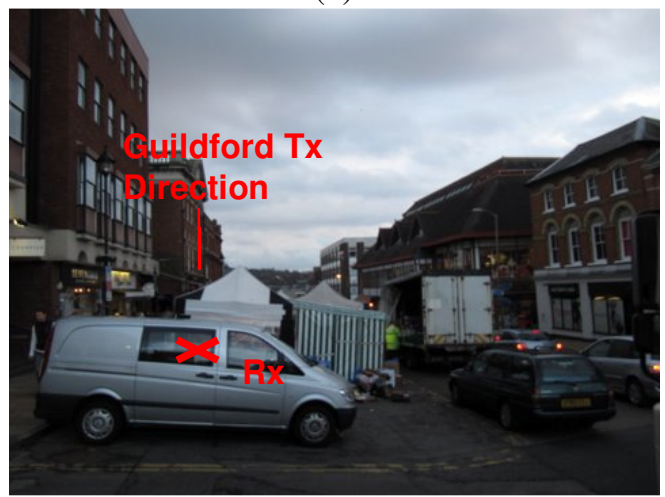

(c)

Figure 1 - Photograph of the three measurement areas chosen: (a) Cathedral, (b) Stoke Park and (c) North Street

An indoor location was not chosen in this measurement campaign. The reason for this was because the likelihood of high de-polarization as well as high penetration loss, thus the majority of television transmissions other than the local ones would fall below the noise floor of the WSD in general and thus in such scenarios interference from the primary user becomes negligible. Therefore only outdoor locations were chosen, where WLAN access points or backhaul links are likely to be exploited. On the day chosen, the weather was dry, with no clouds and sunshine, thus the tropospheric effects were negligible and this would ensure a fair comparison between polarizations measured separately within an approximately ten minute time window over all polarizations and the whole frequency band.

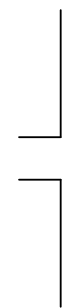

(a)

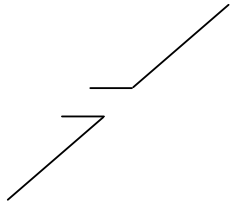

(b)

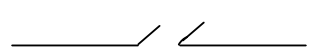

(c)
Figure 2 - Illustration of Dipole Orientations for the (a) vertical polarization, (b) horizontal polarization and (c) horizontal polarization with $90^{\circ}$ re-orientation

To measure vertical and horizontal polarizations, it is necessary to have a polarization pure antenna to measure both vertical and horizontal polarizations over the whole frequency band. Such an antenna is difficult to build with such a wide bandwidth, thus a set of six dipole antennas were used to measure sections of the whole television band at center frequencies of $470 \mathrm{MHz}, 540 \mathrm{MHz}, 590 \mathrm{MHz}, 640 \mathrm{MHz}$, $700 \mathrm{MHz}$ and $750 \mathrm{MHz}$. Each of the antennas were tested for suitable impedance matching and constant gain over the bands they were used for. Since each antenna was used for a separate measurement, three different polarizations were measured one after the other, first of all the dipole was oriented vertically as shown in Figure 2 (a), after which two horizontal polarizations were measured as shown in Figure 2 (b) and (c). The purpose of rotating the antenna $90^{\circ}$ in azimuth in this case is to ensure that if a horizontally polarized signal was arriving at in the same direction as one of the "nulls" in the dipole gain pattern, then the other measurement would pick up such scenarios. This is clearly not allowing a full comparison of the vertical and horizontal polarization, since it is well known that the most suitable way to measure the horizontal polarization omni-directionally is to use a loop antenna [16]. However, the gain of such an antenna is not comparable to that of a dipole and is therefore difficult to calibrate. Thus the purpose of these measurements is to take a "best case scenario" whereby the measurements are comparing the highest vertical polarization with the highest horizontal polarization, which could be received by antennas possible to implement in a WSD. The dipole is the best case scenario because it is more polarization pure than any other practical antenna which could be used on a WSD. This is comparable to polarization diversity systems whereby the polarization aspect is inherent within the angular system [17] and the measurements give an evaluation of whether a WSD with horizontal linear polarization will 
receive less interference from television transmitters, the majority of which are vertically polarized, than that of one with vertical polarization.

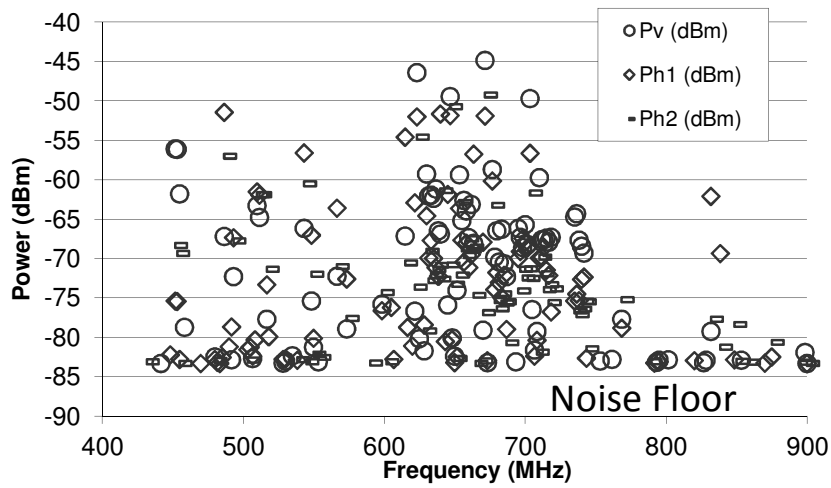

Figure 3 - Example measurements taken for the vertical and two horizontal polarizations

The $\mathrm{dBm}$ power levels measured for the whole television band taken at the cathedral location as an example are compared for all three polarization states shown in Figure 3. Measurements taken below the indicated noise floor threshold (below which is spectrum analyzer noise) of $-86 \mathrm{dBm}$ are omitted. Clearly it can be shown for a number of cases that there are considerably different power levels as high as $10 \mathrm{~dB}$ ratios or more when comparing vertical and horizontal polarizations. In some cases there are higher signals in the horizontal polarization, which would be explained due to such channels coming from the horizontally polarized transmitters while also it is observed that the signals are generally higher between $610 \mathrm{MHz}$ and $720 \mathrm{MHz}$, within which the vertically polarized channels for Guildford are contained and thus demonstrate a stronger vertical polarization.

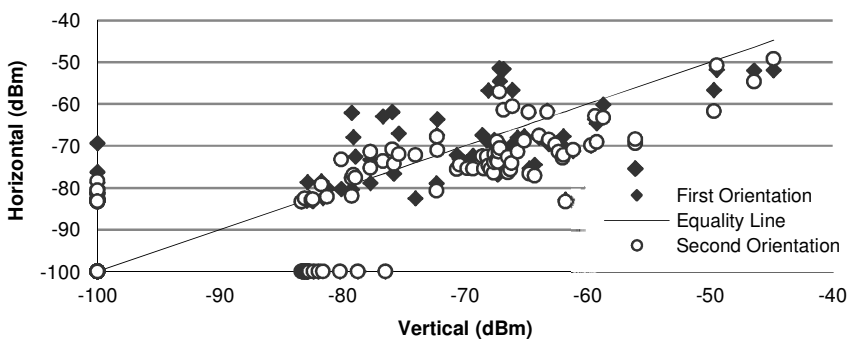

Figure 4 - Scatter plot showing direct comparisons of vertical and horizontal power at fixed frequencies in the Cathedral location

A further useful example comparison to make is to take each frequency and compare the vertical and horizontal polarized power levels on a scatter plot like that in Figure 4 for the Cathedral location. Two separate scatter plots are shown in this case, one where the vertical power is compared with the power of the first horizontal polarization orientation while in the second case, the vertical power is compared with the second horizontal polarization orientation. In the majority of cases, the points are below the line, which makes the horizontal case weaker than the vertical at the same point in space. In cases where either the horizontal or vertical are at a value of $-100 \mathrm{dBm}$, this is where the signal of one of the polarizations fell below the noise floor of the spectrum analyzer and thus no true comparison can be made, though this still occurs more often for horizontal polarization where it is weaker than the vertical.

In order to make an aggregate analysis of all the measured data, a cumulative distribution plot is shown in Figure 5 of the ratio of received vertical power to the received horizontal power for all frequencies. Curves are compared for all three different locations but also for both horizontal polarization orientations. Hence there are six curves to compare given two horizontal polarizations for each location. The following observations can be made from the data presented in Figure 5:

All curves cross the $0 \mathrm{~dB}$ ratio point with a cumulative probability value of $30-35 \%$. This therefore means that for approximately one third of all channels, the vertical polarization is weaker than the horizontal polarization and therefore for approximately two thirds of the channels (i.e. 67\%), the vertical polarization is stronger than the horizontal.

Above 0dB, all the curves roughly overlap each other. Therefore if the point which intersects $6 \mathrm{~dB}$ is taken as shown in Figure 5, it can be interpreted that in $30 \%$ of the channels, the vertical power is $6 \mathrm{~dB}$ higher than the horizontal.

- If a similar analysis was made as with the previous point where the curves intersect $10 \mathrm{~dB}$, then in approximately $10 \%$ of cases, the vertical is $10 \mathrm{~dB}$ higher than the horizontal.

For the case of the first Cathedral measurement, the curve is clearly different from the other curves for cases below $0 \mathrm{~dB}$. This is due to the strong line of sight from the Guildford transmitter, which caused one polarization to be significantly different as a null of the dipole was pointing towards the transmitter.

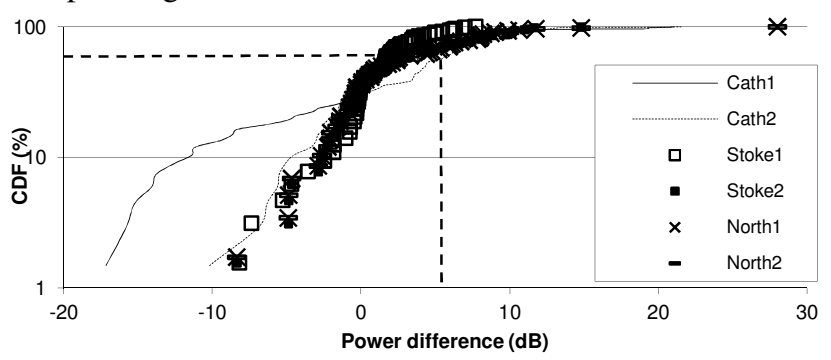

Figure 5 - Cumulative distribution plot of the vertical power to horizontal power aggregated over all frequencies

It is necessary to acknowledge that the results presented here are very specific to the deployment of digital television in the United Kingdom, where the majority of backup transmitters are vertically polarized. In other parts of the world such as the United States, the vast majority of transmitters are horizontally polarized and even in some exceptional cases circular polarization is deployed based on information from the Federal Communications Commission $(\mathrm{FCC})^{2}$. In Australia, information from the Australian Communications and Media

\footnotetext{
${ }^{2}$ http://www.fcc.gov/encyclopedia/tv-query-broadcast-station-search
} 
Authority (AMCA) ${ }^{3}$ also shows a larger number of horizontally polarized deployments. Therefore, if the secondary user is vertically polarized in such countries, it may be less vulnerable to interference from the primary user. However, this is still a separate issue from minimizing the interference from the secondary user to the primary user, which is of more importance and discussed in section IV.

\section{MEASUREMENT OF UdA-Yagi ANTENNA Polarization AND LEAKY FEEDERS}

Before analyzing propagation channels between WSDs and primary receivers in TVWSs, it is important to consider typical Uda-Yagi antennas used in television broadcasting and consider what vulnerability they have to receiving both copolarized and cross-polarized signals at a given angle with the inclusion of their leaky feeders. This can be analyzed by taking the elevation angle measurements of four selected typical example Uda-Yagi antennas illustrated in Figure 6 as follows:

1. An unused high gain wideband Uda-Yagi antenna, which is particularly used in rural locations and has some deliberate increase in cross polarization due to the crossed director elements in Figure 6 (a).

2. A typical low cost receiver antenna was used in Figure 6 (b) in the oldest style, which has undergone significant wear and tear through wind, perching of birds and heat from the sun. It is of interest to identify how such wear and tear has affected its coand cross-polar properties.

3. This antenna is similar to that in 2 though not illustrated in Figure 6. The purpose of measuring this antenna is to compare two antennas to identify the typical effect of wear and tear and see if it is typical between antennas.

4. A high gain wideband antenna but with a deliberately leaky feed, where the cable has been taped to the feed in an ad-hoc fashion shown in Figure 6 (c) as may be the case if installed by a home owner with less than amateur knowledge of how to install the antenna, though still able to allow the antenna to function for a television receiver. The impact on cross polarization was necessary to analyze.

All antennas were tested in an anechoic chamber at a frequency of $530 \mathrm{MHz}$, due to the limited bandwidth of the two antennas with significant wear and tear, in which there was over $10 \mathrm{~dB}$ return loss, while the bandwidth of the other two antennas was significantly wider. Their principal elevation patterns were measured, both co-polar and cross-polar. It should be noted in this case that a short coaxial cable of $75 \Omega$ of less than $2 \mathrm{~m}$ was connected to the antenna, followed by a $75 \Omega$ to $50 \Omega$ conversion in order to measure the gain pattern. The cable length and conversion loss does reduce the gain of the antenna measured, though such loss compared to what would result from the length of cable that would reach a television receiver with a leaky feeder is small and thus a maximum possible gain of the antenna and cable is measured, while a direct comparison between co polar and cross polar gains can be made.

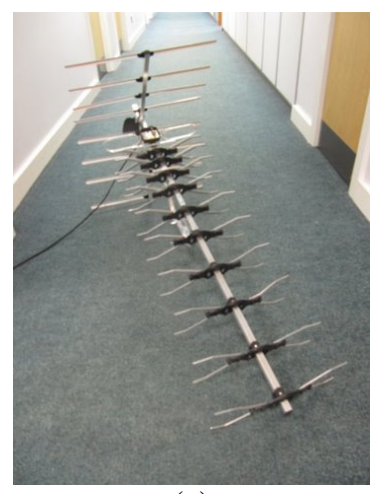

(a)

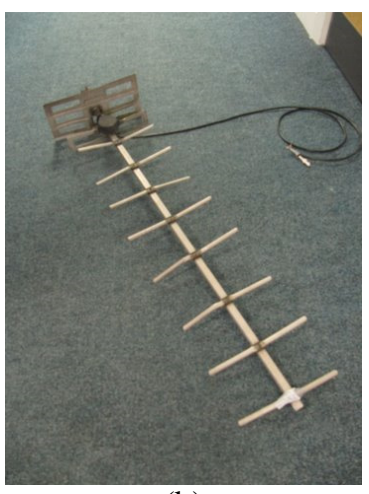

(b)

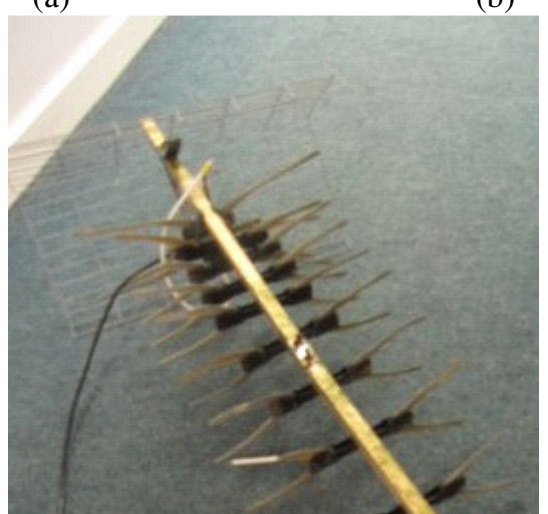

(c)

Figure 6 - Illustration of the different types of antenna used as example cases (a) new wideband antenna (b) old antenna (two tested for repeatability) and (c) antenna with deliberately leaky feeder

Two example cases of the antenna patterns are shown in Figure 7 measured at $530 \mathrm{MHz}$, where it is seen that the coand cross-polar gains are comparable when receiving from the rear and side ends of the antenna, while towards the boresight, there is nearly $10 \mathrm{~dB}$ cross polarization rejection for the old antenna number 1 in Figure 7 (a). If the feeders are made deliberately leaky, this does not actually degrade the co-polar gain that significantly though the cross polarization does increase and is not necessarily in the direction of boresight as illustrated in Figure 7 (b). The cross polar gain is over $3 \mathrm{dBi}$ maximum, which is better gain than an ideal dipole $(2.1 \mathrm{dBi})$ and thus susceptible to receiving fields in that polarization. The $3 \mathrm{~dB}$ beamwidths of the antennas are around $30^{\circ}$ as is typically expected for Uda-Yagi antennas.

The maximum co-polar and cross-polar gain values are compared in Table 2, where it can be seen that the new antenna having wide bandwidth has a lower gain than the old antennas, which have around $9 \mathrm{~dB}$ cross polarization rejection. Given that this reduces to $3 \mathrm{~dB}$ with deliberately leaky feeders, the antennas alone cannot be relied upon to significantly reduce interference based on their polarization alignment. 


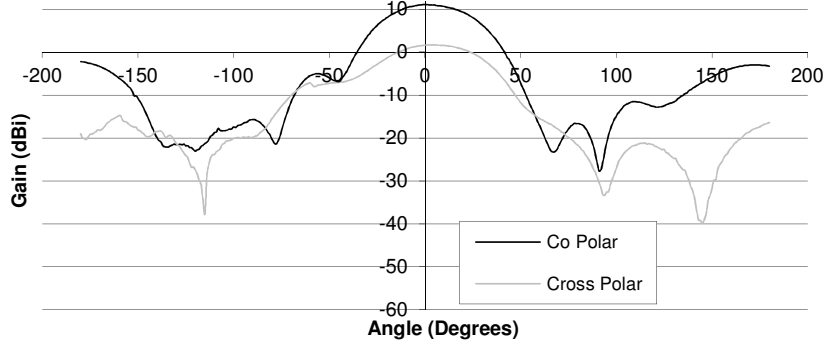

(a)

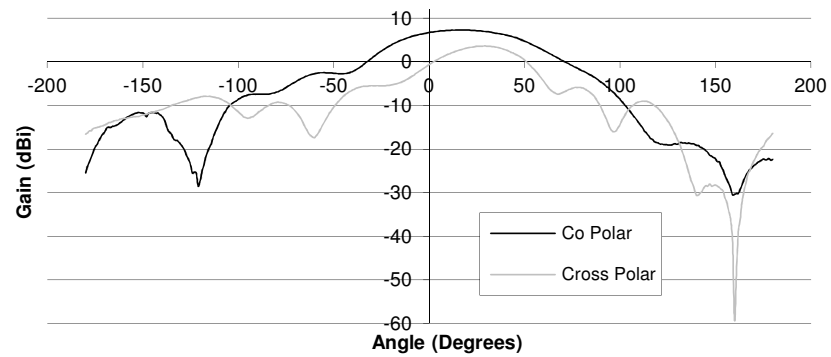

(b)

Figure 7 - Comparison of co-polar and cross polar antenna patterns from (a) old antenna 1 and (b) antenna with deliberately leaky feeders

\begin{tabular}{|l|c|c|}
\hline \multicolumn{1}{|c|}{ Antenna } & $\begin{array}{c}\text { Max Co- } \\
\text { Polar } \\
\text { Gain }(\mathbf{d B i})\end{array}$ & $\begin{array}{c}\text { Max Cross } \\
\text { Polar Gain } \\
(\mathbf{d B i})\end{array}$ \\
\hline New Antenna & 7.28 & -0.11 \\
\hline Old Antenna 1 & 11.13 & 1.70 \\
\hline Old Antenna 2 & 8.50 & -0.45 \\
\hline Deliberately Leaky & 7.83 & 3.68 \\
\hline
\end{tabular}
Table 2 - Maximum Co-polar and Cross-polar gain values
for the tested Uda-Yagi antennas

\section{PATH LOSS ANALYSIS ON INTERFERENCE TO PRIMARY USER}

Traditionally path loss has been measured and modeled with the purpose of ensuring there is sufficient coverage in a given area from a transmitter or base station so that a suitable fade margin can be chosen to ensure sufficient coverage depending on any further un-predictable penetration losses or impact due to how the receiver antenna is used or orientated. Therefore the path loss and the addition of a fade margin considers a maximum loss case so as to ensure coverage, while in the case of considering the path loss from a WSD to a television receiver or re-transmitter, it is necessary to consider what the minimum path loss would be and thus ensure a range beyond which there is not interference to the primary user. A further novelty is therefore useful if the polarization of the WSD is able to significantly increase the path loss from the WSD to the primary user, while also minimizing path loss between WSDs communicating with each other. This section therefore analyses through measurement how polarization can help in this endeavor.

For simplicity in deploying WSDs, it could be argued that the free space path loss model is suitable to use for evaluating the maximum potential interference from the primary user to the secondary user. The grounds for such an argument, if the antenna gains at each end were known, would be that the cable loss, scattering losses and any other imperfections would cause this loss to be exceeded. Such a model, though simple, would prove restrictive in the deployment of WSDs because the power levels permitted to be used by them will be too low for purpose. Therefore it is necessary to consider a model that is more realistic and closer to reality so it will give more deployable ranges over which WSDs can transmit. One such model that can be considered is the well known plane earth loss (PEL) or two path model approximated as follows [18]:

$$
\mathrm{PEL}=\frac{P_{\mathrm{Rx}}}{P_{\mathrm{Tx}}} \approx\left(\frac{\lambda}{4 \pi r}\right)^{2}\left|1-e^{-\mathrm{j} \beta \frac{2 h_{\mathrm{WSD}} h_{\mathrm{PU}}}{r}}\right|^{2} \approx \frac{h_{\mathrm{WSD}}^{2} h_{\mathrm{PU}}^{2}}{r^{4}}
$$

where $\beta$ is the phase constant, $\lambda$ is the wavelength, $h_{\mathrm{WSD}}$ and $h_{\mathrm{PU}}$ are the respective antenna heights of the white space device and the primary user. Note that path loss used in this paper is a ratio of received power, $P_{\mathrm{Rx}}$ to transmit power $P_{\mathrm{Tx}}$, thus the calculated values will be less than unity or $0 \mathrm{~dB}$. An important assumption in this model is that the electric E-field path reflected off the ground has a low grazing angle but also has a phase inversion. Therefore with high values of $r$, the two E-fields from the two paths will arrive at the receiver almost in anti-phase. This does assume also that the wavelength at the frequency of operation is several times higher in magnitude than the difference in distance between the two paths. Therefore the approximation in equation (1), which is independent of frequency, is only valid below a maximum frequency, but also beyond a minimum distance $r$.

The assumption of the reflection in anti-phase does assume that the transmitted E-field is parallel to the ground (i.e. horizontally polarized) according to Fresnel's equations [19]. However, if the E-field is vertically polarized then it will be almost normal to the ground when the grazing angle is low and the reflection will be in phase, thus consistent with the boundary conditions for such a case. Therefore in the ideal case with no other scattering components, if there is vertical polarization, low enough frequency and high enough distance $r$, then the approximation for the PEL in the vertical case (as a ratio of vertically polarized received to transmitted power) is derived as follows:

$$
\mathrm{PEL}_{\mathrm{V}}=\frac{P_{\mathrm{Rx} I \mathrm{~V}}}{P_{\mathrm{Tx} I \mathrm{~V}}} \approx\left(\frac{\lambda}{4 \pi r}\right)^{2}\left|1+e^{-\mathrm{j} \beta \frac{2 h_{\mathrm{WSD}} h_{\mathrm{PU}}}{r}}\right|^{2} \approx 4\left(\frac{\lambda}{4 \pi r}\right)^{2}
$$

Therefore the path loss is $6 \mathrm{~dB}$ lower than free space loss (i.e. $6 \mathrm{~dB}$ less attenuation). In many practical cases for terrestrial communication there is clutter present and even one nearby scatterer will cause a path in anti-phase as in the case of horizontal polarization, because the vertical surface of the scatterer will then be parallel with the vertically polarized E- 
field. For this reason, in many terrestrial communications the model in equation (1) is widely accepted for both polarizations and also measurements carried out in urban environments at low heights at the UHF band [20] where polarization was not a factor. However, for the case of TVWSs, where the WSD is acting as a super WiFi access point or backhaul link, it will be positioned above the clutter in many cases as will the UdaYagi antenna connected to the primary user. Furthermore at low heights above the clutter, the grazing angle will be low and the clutter can be assumed to have a low surface roughness according to the Rayleigh criterion [18]. In such cases above the surface of the clutter or even where there is a minimal amount of clutter, it is arguable that horizontal polarization is necessary to increase the path loss and be more comparable with that of PEL, or even greater as the outdoor experiment documented in the next sub section explains, which is further complemented by the indoor measurement carried out in the sub section beyond.

\section{A. Outdoor Path Loss Measurement}

Given the constraints involved in terms of using licensed spectrum, an outdoor path loss measurement was set up as illustrated in Figure 8 for purposes of measuring the impact of polarization on the path loss, while best representing the scenario of a WSD interfering with a television receiver as though it was placed above the height of buildings or that they were situated over widely open terrain. The new wideband Uda-Yagi antenna in Figure 6 (a) was used as the receive antenna, acting as the primary user, set on a mast at a height of $1.04 \mathrm{~m}$ and was connected to a spectrum analyzer, which had a minimal resolution and video bandwidth, with a span of $200 \mathrm{kHz}$ to ensure that a measurement with maximum sensitivity could be taken. A half wavelength dipole antenna, representing the WSD, was attached to a non-metallic pipe to keep it a distance away from the trolley but close to the ground at a height of $0.22 \mathrm{~m}$. It was also possible to switch the polarization of both the Uda-Yagi and the dipole from vertical to horizontal to take measurements of path loss between different polarizations. The dipole was connected to a signal generator with low transmit power below $-40 \mathrm{dBm}$. This was necessary because it consequently meant that beyond the open space (which accommodated a radial distance of at least $15 \mathrm{~m}$ ) the equivalent isotropic radiated power (EIRP) at that distance would be below the maximum allowable EIRP within the ultra wideband (UWB) spectrum mask, which is $-90 \mathrm{dBm}$ at UHF frequencies in the United Kingdom ${ }^{4}$. A frequency of $610 \mathrm{MHz}$ was chosen because it was an undetected broadcasting channel at the measurement location and thus would not cause interference to the measurement.

The more specific dimensions of the measurement setup are illustrated in Figure 9 and Figure 10, which also show that in this measurement scenario, there are not only the first two paths from the line of sight (not labeled as the first path for clarity) and the reflection off the ground (labeled as the second

\footnotetext{
${ }^{4}$ Regulated by Office for Communications, www.ofcom.org.uk
}

path), but an additional two paths, which reflect off the trolley at large distances (noted as the third and fourth paths). These four paths all have their respective distances (including the distance from the Uda-Yagi antenna, to the reflectors and the dipole antenna), $r_{1}, r_{2}, r_{3}$ and $r_{4}$. As the Uda-Yagi antenna is representing the primary user it has height $h_{\mathrm{PU}}$ and as the dipole antenna represents the WSD so it has height $h_{\mathrm{WSD}}$ separated by distance $r$.

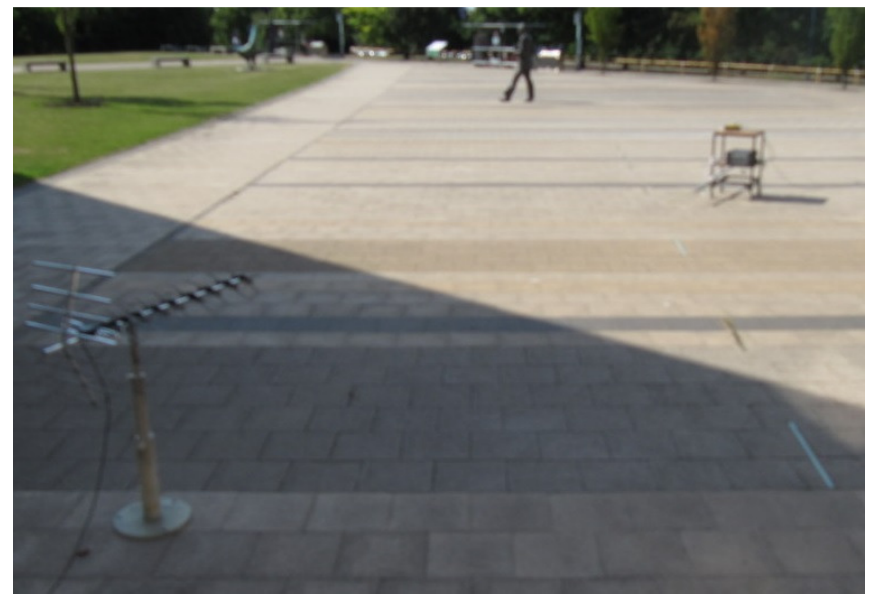

(a)

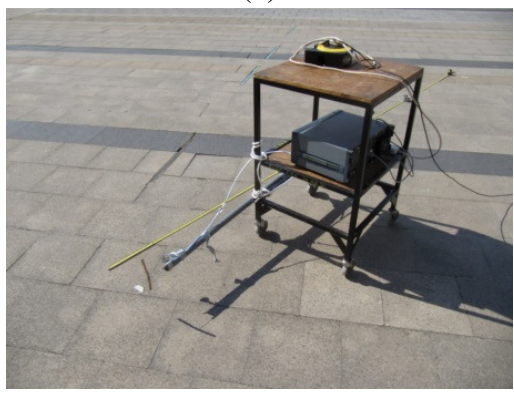

(b)

Figure 8 - Illustration of the outdoor path loss measurement set up showing (a) the open environment and (b) the arrangement of the dipole on the trolley

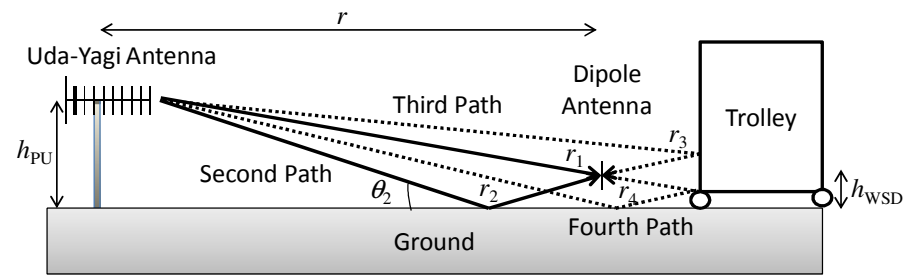

\section{Figure 9 - Illustration of the four paths encountered in the measurement scenario}

For purposes of comparing measured results with the theoretical four path model in this case, it is first necessary to establish the lengths of the four path distances, $r_{1}, r_{2}, r_{3}$ and $r_{4}$ based on knowing the transmit and receive antenna heights $h_{\mathrm{PU}}$ and $h_{\mathrm{WSD}}$ as well as the distance $r$. For the first two paths, it is well known that the distances can be derived based on the three given distances using Pythagoras' theorem as follows 
[18]:

$$
\begin{aligned}
& r_{1}=\sqrt{\left(h_{\mathrm{PU}}-h_{\mathrm{WSD}}\right)^{2}+r^{2}} \\
& r_{2}=\sqrt{\left(h_{\mathrm{PU}}+h_{\mathrm{WSD}}\right)^{2}+r^{2}}
\end{aligned}
$$

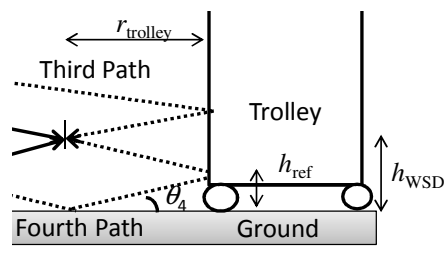

\section{Figure 10 - Close up illustration of the four paths arriving} at the dipole next to the trolley

The third path can be calculated in a similar manner provided the distance from the dipole to the trolley, $r_{\text {trolley }}$, illustrated in Figure 10 is known. In this measurement, the value of $0.98 \mathrm{~m}$ was used and the equation is derived as follows (assuming the trolley is high enough to cause a reflection, which was so in this case):

$$
r_{3}=\sqrt{\left(h_{\mathrm{PU}}-h_{\mathrm{WSD}}\right)^{2}+\left(r+2 r_{\text {trolley }}\right)^{2}}
$$

For calculating the distance for the fourth path, it is necessary to know the height at which it reflects of the trolley, $h_{\text {ref }}$, as illustrated in Figure 10. The equation can be derived by knowing its relation to the grazing angle of the fourth path, $\theta_{4}$ illustrated in Figure 10, which relates to $h_{\mathrm{ref}}$ by the following two trigonometric relations:

$$
\begin{aligned}
& \tan \theta_{4}=\frac{h_{\mathrm{PU}}+h_{\mathrm{ref}}}{r+r_{\text {trolley }}} \\
& \tan \theta_{4}=\frac{h_{\mathrm{WSD}}-h_{\mathrm{ref}}}{r_{\text {trolley }}}
\end{aligned}
$$

Hence these two simultaneous equations can resolve $h_{\text {ref }}$ to be:

$$
h_{\text {ref }}=\frac{h_{\mathrm{wSD}}\left(r+r_{\text {trolley }}\right)-h_{\mathrm{PU}} r_{\text {trolley }}}{r+2 r_{\text {trolley }}}
$$

Hence the distance $r_{4}$ can be derived as follows:

$$
\begin{aligned}
r_{4}= & \sqrt{\left(h_{\mathrm{PU}}+h_{\text {ref }}\right)^{2}+\left(r+r_{\text {trolley }}\right)^{2}} \\
& +\sqrt{\left(h_{\mathrm{WSD}}-h_{\text {ref }}\right)^{2}+r_{\text {trolley }}^{2}}
\end{aligned}
$$

Having established the path lengths $r_{1}$ to $r_{4}$, the path differences used in model comparing the distance of the second, third and fourth paths to the line of sight, $\Delta r_{2}$ to $\Delta r_{4}$, are defined as follows:

$$
\begin{aligned}
\Delta r_{2} & =r_{2}-r_{1} \\
\Delta r_{3} & =r_{3}-r_{1} \\
\Delta r_{4} & =r_{4}-r_{1}
\end{aligned}
$$

Using the same approach as for the two path model [18], only this time extending it to four paths from two, the vertically and horizontally polarized path loss (PL) equations are derived for any distance $r$ as follows (assuming $\Delta r_{2}, \Delta r_{3}, \Delta r_{4}<<r$ ):

$$
\begin{aligned}
\mathrm{PL}_{4 \mathrm{PathlV}} & =\frac{P_{\mathrm{RxIV}}}{P_{\mathrm{TxlV}}} \\
& =\left(\frac{\lambda}{4 \pi r}\right)^{2}\left|1+R_{\mathrm{V}}\left(\theta_{2}\right) e^{\mathrm{j} \beta \Delta \Delta_{2}}-e^{\mathrm{j} \beta \Delta_{3}}-R_{\mathrm{V}}\left(\theta_{4}\right) e^{\mathrm{j} \beta \Delta_{4}}\right|^{2} \\
\mathrm{PL}_{4 \mathrm{PathlH}} & =\frac{P_{\mathrm{Rx|H}}}{P_{\mathrm{TxH}}} \\
& =\left(\frac{\lambda}{4 \pi r}\right)^{2}\left|1+R_{\mathrm{H}}\left(\theta_{2}\right) e^{\mathrm{j} \beta \Delta \Delta_{2}}-e^{\mathrm{j} \beta \Delta \Delta_{3}}-R_{\mathrm{H}}\left(\theta_{4}\right) e^{\mathrm{j} \beta \Delta \Delta_{4}}\right|^{2}
\end{aligned}
$$

The grazing angle for the second path, $\theta_{2}$ is illustrated in Figure 9. Both grazing angles, $\theta_{n}$ are used to calculate the vertical and horizontal ground reflection coefficients, $R_{\mathrm{V}}$ and $R_{\mathrm{H}}$ as follows [19]:

$$
\begin{aligned}
& R_{\mathrm{V}}\left(\theta_{n}\right)=\frac{\sqrt{\varepsilon_{r}-\cos ^{2} \theta_{n}}-\varepsilon_{r} \sin \theta_{n}}{\sqrt{\varepsilon_{r}-\cos ^{2} \theta_{n}}+\varepsilon_{r} \sin \theta_{n}} \\
& R_{\mathrm{H}}\left(\theta_{n}\right)=\frac{\varepsilon_{r} \sin \theta_{n}-\sqrt{\varepsilon_{r}-\cos ^{2} \theta_{n}}}{\varepsilon_{r} \sin \theta_{n}+\sqrt{\varepsilon_{r}-\cos ^{2} \theta_{n}}}
\end{aligned}
$$

where $\varepsilon_{r}$ is the dielectric constant of the ground, assumed to have the value of 6 in these measurements (though its value makes negligible difference to the result). It can be seen clearly that as the grazing angle tends to zero, $R_{\mathrm{V}}$ tends to 1 while $R_{\mathrm{H}}$ tends to -1 . Using equations (13) and (14) for conductors, regardless of polarization and grazing angles except for angles close to $90^{\circ}$ (which are not used here), the reflection off the metallic trolley is always -1 , thus the third path reflection coefficient is -1 in equations (11) and (12) and the fourth path's reflection coefficient is inverted since it includes a reflection off the trolley. The polarity of the reflection coefficients therefore changes due to the ground reflections only and whatever polarization is incident on the ground. The following can be inferred from equations (11) and (12) when the distance $r$ is large, thus $R_{\mathrm{V}}$ and $R_{\mathrm{H}}$ are +1 and -1 respectively:

- For vertical polarization, the first path is almost out of phase with the third path if $\Delta r_{3}$ is small, thus the 
two nearly cancel out. The second and fourth paths also result in being almost out of phase if $\Delta r_{2}$ and $\Delta r_{4}$ are small, while also $\theta_{4}$ and $\theta_{2}$ are almost the same.

- For horizontal polarization, the first and second path are closer to the point of anti-phase than any other combination of paths. The third and fourth path, however, are still near to anti phase. This distinct difference of the first and second path, compared to the vertical polarization, means that the path loss is greater for horizontal polarization.

To evaluate this difference, the measurement results from the path loss are compared with the theoretical predictions in equations (11) and (12), as well as the plane earth loss predictions in equation (1) plotted in Figure 11. Note, however that in this case the gains of the two antennas, denoted $G_{\text {Dipole }}$ and $G_{\text {Uda-Yagi }}$ have been factored in to allow for fair comparison. The free space path loss, with antenna gains also plotted, is defined as follows [16]:

$$
\mathrm{PL}_{\text {FreeSpace }}=\frac{P_{\text {TxlFree Space }}}{P_{\text {RxlFree Space }}}=G_{\text {Uda-Yagi }} G_{\text {Dipole }}\left(\frac{\lambda}{4 \pi r}\right)^{2}
$$

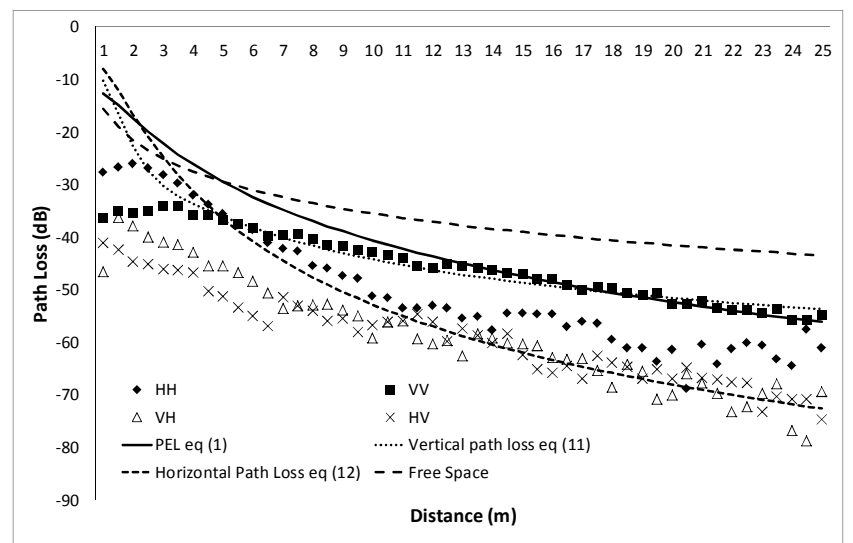

Figure 11 - Comparison of the measured and predicted path loss in the outdoor environment

Four measurements are shown, where $1 \mathrm{~m}$ steps are taken of the vertical to vertical (VV), horizontal to horizontal ( $\mathrm{HH})$ copolar cases; the vertical to horizontal $(\mathrm{VH})$ and the horizontal to vertical (HV) cross-polar cases. For the VV case it is in good agreement with the theoretical predictions from $6 \mathrm{~m}$ and beyond. This is expected because at shorter than this distance, the signal is received outside the Uda-Yagi antenna's beamwidth and thus there is a change in antenna gain. Furthermore the four path model is not valid at distances below this value. It is interesting to note that the vertically polarised four path model becomes comparable with the horizontally polarised two path model at large distances beyond $17 \mathrm{~m}$. It could be assumed given this similarity that the two path PEL model is acceptable for both polarizations but it is not modelling the actual case as evidenced by the difference at shorter distances, while the four path model is in better agreement.
The HH measurements are clearly showing a greater path loss than the VV case, as is expected from the theoretical predictions, though the measured case does not show as strong agreement with the predicted horizontal path loss. This can be explained due to the roughness of the ground, which means the anti-phase between the first two paths as well as the third and fourth paths is not as close as the theoretical, thus the loss is reduced but nonetheless still the loss is greater, in the order of $10 \mathrm{~dB}$ in a number of measured points. Therefore scenarios on top of buildings and open terrain representative of WSD to primary user links, with low clutter in the vicinity, horizontal polarization is a better choice for the WSD because it will result in a larger path loss as desired. Comparing the co-polar and cross-polar cases in Figure 11, it could be argued that the WSD should always use the opposite polarization to the primary user to gain a greater path loss. However, this will not hold if the antenna has leaky feeders and section III has shown this potential vulnerability. Therefore horizontal polarization from the WSD will still ensure a greater minimum path loss is achieved should the primary user antenna be defective.

\section{B. Indoor Path Loss Measurement}

The same measurement setup in terms of heights and distances was also carried out indoors in a corridor, but in this instance the transmitted power was lower below $-50 \mathrm{dBm}$ in order to comply with transmission regulations.

The results are plotted in Figure 13, where in this instance it is too complex to create predicted ray models. The purpose of these measurements is to clarify that at large distances, beyond $23 \mathrm{~m}$ in this case, there are some instances where a greater path loss is found in $\mathrm{HH}$ compared to $\mathrm{VV}$, which is expected because the line of sight and ground reflected path become more dominant at such distances in comparison to the other scattered rays. Hence the major anti-phase from the ground reflection in horizontal polarization can still have some effect when high clutter is present. Taking the cross polar measurements, $\mathrm{VH}$ and $\mathrm{HV}$, they have comparable benefits to the outdoor measurement case.

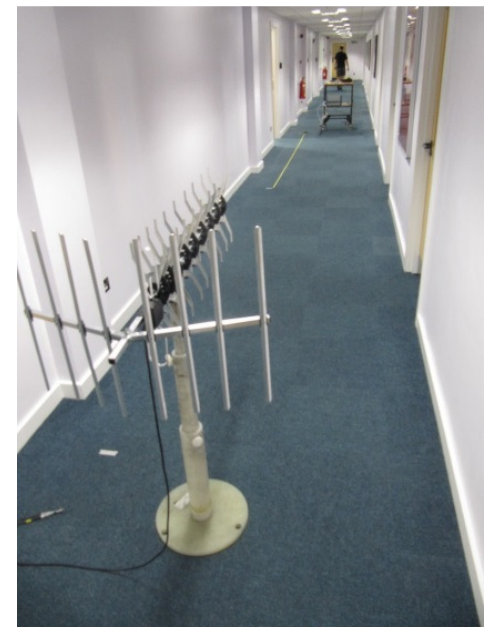

Figure 12 - Illustration of the indoor path loss measurement setup 


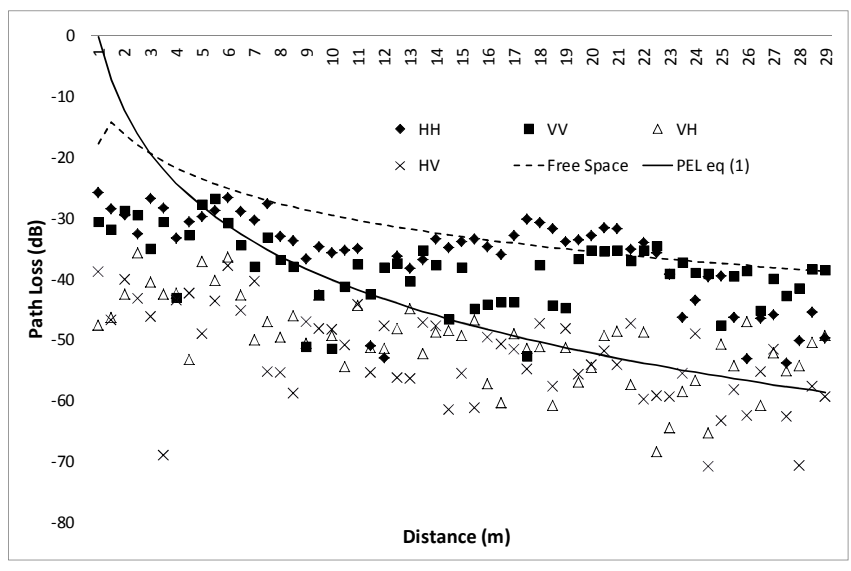

Figure 13 - Results of the measured path loss for the indoor environment against free space and PEL models

As a final important note to these measurements, it should be addressed that consideration of obstructions to the line of sight causing diffraction or shadowing were not considered. For interference from the WSD to the primary user, the worst case scenario is of interest, where there are no obstructions and thus it is of interest to find out what is the minimum guaranteed path loss between the WSD and the transmitter. Clearly horizontal polarization increases the chance of this path loss exceeding a minimum value compared to vertical polarization and hence any diffracting objects will increase this path loss further beyond the minimum as opposed to decreasing it. Such factors are of less interest however when considering the safe deployment of WSDs.

\section{CONCLUSION}

Measurements have been presented in this paper, which show the benefit of using horizontal polarization in WSDs deployed at high heights. Where the majority of broadcasting transmitters are vertically polarized, this can reduce interference to the WSD but conversely, it is necessary to use dual polarization to avoid errors in spectrum sensing. Regardless of the polarization of the primary user, an increase in path loss from the WSD to the primary user is assured if the WSD has horizontal polarization. The path loss measurements and analysis also show that the widely used two path plane earth loss model is only valid for horizontal polarization and though it may appear to be consistent with measurements taken in vertical polarization it is not representative of the actual propagation phenomena, where in these measurements a four path model verified. In the case where there are few scatterers in the propagation environment, as would be true in a worst case scenario for WSDs, the path loss can be further increased beyond the PEL case, which is beneficial in reducing interference to the primary user.

\section{REFERENCES}

[1] M. Nekovee, "Cognitive Radio Access to TV White Spaces: Spectrum Opportunities, Commercial Applications and Remaining Technology Challenges", IEEE Symposium in New Frontiers in Dynamic Spectrum, pp1-10, April 2010.

[2] D. Noguet, R. Datta, P. H. Lehne, M. Gautier, G. Fettweis, "TVWS regulation and QoSMOS requirements", International Conference on
Wireless Communication, Vehicular Technology, Information Theory and Aerospace \& Electronic Systems Technology, 2011.

[3] W. Webb, "On Using White Space Spectrum", IEEE Communications Magazine, vol. 50, issue 8, pp145-151, August 2012.

[4] M. Fitch, M. Nekovee, S. Kawade, K. Briggs, R. MacKenzie, "Wireless Service Provision in TV White Space with Cognitive Radio Technology: A Telecom Operator's Perspective and Experience", IEEE Communications Magazine, vol. 49, issue 3, pp64-73, 2011.

[5] M. Waddell, T. Harrold, "Measured DVB-T Protection Ratios in the presence of Interference from White Space Devices", British Broadcasting Corporation White paper, WHP226, April 2012.

[6] M. Nekovee, "Quantifying the Availability of TV White Spaces for Cognitive Radio Operation in the UK", IEEE International Comnference on Communications, pp1-5, 2009.

[7] D. Gurney, G. Buchwald, L. Ecklund, S. Kuffner, J. Grosspietsch, "Geolocation Database Techniques for Incumbent Protection in the TV White Space", IEEE Symposium on New Frontiers in Dynamic Spectrum Access Networks, pp1-9, 2008

[8] M. Denkovska, P. Latkoski, L. Gavrilovska, "Geolocation Database Approach for Secondary Spectrum Usage of TVWS", Telecommunications Forum, pp369-372, 2011.

[9] V. Gonçalves, S. Pollin, "The value of sensing for TV White Spaces", IEEE International Symposium on New Frontiers in Dynamic Spectrum Access Networks, pp231-241, May 2011.

[10] H-S Chen, W. Gao, "Spectrum Sensing for TV White Space in North America", IEEE Journal on Selected Areas in Communications, vol. 29, no. 2, pp316-326, 2011.

[11] S. J. Shellhammer, "A Comparison of Geo-Location and Spectrum Sensing in Cognitive Radio", International Conference on Computer Communications and Networks, pp1-6, 2009.

[12] D. Borth, R. Ekl, B. Oberlies, S. Overby, "Considerations for Successful Cognitive Radio Systems in US TV White Space", IEEE Symposium on New Frontiers in Dynamic Spectrum Access Networks, pp1-5, 2008.

[13] D. Noguet, M. Gautier, V. Berg, "Advances in opportunistic radio technologies for TVWS", EURASIP Journal on Wireless Communications and Networking, vol. 170, November 2011.

[14] H. R. Karimi, "Geolocation databases for white space devices in the UHF TV bands: Specification of maximum permitted emission levels", IEEE Symposium on New Frontiers in Dynamic Spectrum Access Networks, pp443-454, May 2011.

[15] G. W. Collins, "Fundamentals of Digital Television Transmission", 2001, Wiley

[16] C. A. Balanis, “Antenna Theory and Design”, Wiley, 2005.

[17] T. W. C. Brown, S. R. Saunders, S. Stavrou and M. Fiacco, "Characterization of Polarization Diversity at the Mobile", IEEE Transactions on Vehicular Technology, vol. 56, issue 5, part 1, pp24402447, September 2007.

[18] S. Saunders, A. Aragón-Zavala, "Antennas and Propagation for Wireless Communication Systems", $2^{\text {nd }}$ Edition, 2007, Wiley.

[19] C. A. Balanis, "Advanced Engineering Electromagnetics", 1989, Wiley.

[20] B. Belloul, "Predicting path loss between terminals of low height", Phase 2, Final Report, Red-M Ltd., 2007. 